Case Report

\title{
Acquired Vitamin K Deficiency as Unusual Cause of Bleeding Tendency in Adults: A Case Report of a Nonhospitalized Student Presenting with Severe Menorrhagia
}

\author{
Omid Reza Zekavat, ${ }^{1}$ Gholamreza Fathpour, ${ }^{1}$ Sezaneh Haghpanah, ${ }^{1}$ \\ Seyed Javad Dehghani, ${ }^{1}$ Maryam Zekavat, ${ }^{2}$ and Nader Shakibazad ${ }^{1}$ \\ ${ }^{1}$ Hematology Research Center, Shiraz University of Medical Sciences, Shiraz, Iran \\ ${ }^{2}$ Department of Biological Engineering, Massachusetts Institutes of Technology, Cambridge, MA, USA \\ Correspondence should be addressed to Nader Shakibazad; nshakibazad@gmail.com
}

Received 15 March 2017; Revised 18 June 2017; Accepted 30 July 2017; Published 27 August 2017

Academic Editor: Irene Hoesli

Copyright (C) 2017 Omid Reza Zekavat et al. This is an open access article distributed under the Creative Commons Attribution License, which permits unrestricted use, distribution, and reproduction in any medium, provided the original work is properly cited.

\begin{abstract}
We report a rare case of acquired vitamin K deficiency presenting with severe menorrhagia and without any gynecological problem. Partial thromboplastin time (59.2 seconds) and prothrombin time (33.1 seconds, INR: 5.97) were considerably prolonged in laboratory evaluations. A complete coagulation factor assay test was performed for the patient: factor IX, $24 \%$; factor II, $41 \%$; factor VII, 3\%; and factor X, 52\%. She had been taking many high-energy drinks and she had inadequate dietary intake for the past 6 months. Given that she had vitamin K deficiency (VKD), a course of vitamin K therapy was started for her in the hospital. This case showed the potential for menorrhagia due to VKD with use of high-energy drinks and the value of a complete and detailed history in early diagnosis.
\end{abstract}

\section{Introduction}

Menorrhagia is a very common clinical condition reported in women of reproductive age. More than half of women with a normal gynecological evaluation will have laboratory abnormalities indicating hemostasis [1]. Menorrhagia can be the first manifestation of a bleeding disorder [2]. The estimated incidence of bleeding disorders as a cause of menorrhagia can be as high as 10-20\% [1]. Coagulopathies are not usually suspected in the etiology of menorrhagia and surgical interventions are done without investigation for coagulopathies [3]. Normal individual with acquired vitamin $\mathrm{K}$ deficiency (VKD) as a cause of menorrhagia is rare, because of small daily requirement of vitamin $\mathrm{K}$ [4].

It is essential for physicians to perform logical clinical and laboratory assessments for an underlying bleeding disorder after gynecological causes are ruled out. Here, we report a rare case of acquired transient VKD as a cause of menorrhagia that is easily preventable in nonhospitalized patient.

\section{Case}

A 23-year-old healthy female consulted with hematologist for the condition of menorrhagia (heavy menstrual bleeding) indicated by the fact that she had to change her pad every 3-4 hours for duration of 3 months. She was teacher and student of master degree of psychiatry. She had been working for long periods of time without any rest for a year. For a period of 4 months prior to seeking medical advice, she had taken an excess of acetaminophen due to body pain. She did not have any past medical history or family history of bleeding tendency or a history of drugs (warfarin or antibiotic). On physical examination, she was only pale. Also, she had no gynecological problem or history of weight loss.

She was evaluated for possible cause of bleeding tendency. Her liver function tests, collagen-vascular disease tests, and thyroid function tests were normal (Table 1). Celiac panel tests also were normal.

In her laboratory work-up, hemoglobin was low $(10.9 \mathrm{~g} / \mathrm{dl})$ and leukocyte count $\left(5.4 \times 10^{3}\right.$ cell $\left./ \mu \mathrm{L}\right)$ and 
TABLE 1: Laboratory findings in our case report.

\begin{tabular}{lcc}
\hline & Result & Unit \\
\hline Liver function tests & & \\
AST & 17 & $\mathrm{U} / \mathrm{L}$ \\
ALT & 15 & $\mathrm{U} / \mathrm{L}$ \\
Total bilirubin & 0.48 & $\mathrm{Mg} / \mathrm{dL}$ \\
Direct bilirubin & 0.17 & $\mathrm{Mg} / \mathrm{dL}$ \\
Albumin & 4 & $\mathrm{~g} / \mathrm{dL}$ \\
Alkaline phosphatase & 110 & $\mathrm{U} / \mathrm{L}$ \\
\hline Collagen-vascular disease & & \\
tests & & \\
ANA & Negative & \\
anti-dsDNA & Negative & \\
ACLA & Negative & \\
APLA & Negative & \\
\hline Thyroid function tests & & $\mathrm{nIU} / \mathrm{ml}$ \\
TSH & 1.97 & $\mathrm{nmol} / \mathrm{L}$ \\
T4 & 102.70 & \\
T3 & 1.27 & \\
\hline
\end{tabular}

AST: aspartate aminotransferase, ALT: alanine aminotransferase, ANA: antinuclear antibody, anti-dsDNA: anti-double-stranded DNA, ACLA: anticardiolipin antibodies, APLA: antiphospholipid antibodies, TSH: thyroidstimulating hormone, and T4: thyroxine.

platelet count $\left(268 \times 10^{3}\right.$ cell $\left./ \mu \mathrm{L}\right)$ were normal (Sysmex KX$\left.21 \mathrm{~N}^{\mathrm{TM}}\right)$; but in evaluation of the coagulation system, bleeding time was prolonged ( 9 minutes, 11 seconds) (IVY method). Also partial thromboplastin time (PTT: 59.2 seconds) and prothrombin time (PT) were significantly prolonged (PT: 33.1 seconds, INR: 5.97$)$ which were corrected in a mixing test without incubation time. Von Willebrand factor (vWF) antigen, vWF activity, and fibrinogen level were normal, and only mild factor $\mathrm{X}$ deficiency $(8 \%)$ had been detected.

Due to iron deficiency signs and symptoms and ferritin $33.3 \mathrm{ng} / \mathrm{mL}$, a course of iron therapy $(4 \mathrm{mg} / \mathrm{kg} /$ day $)$ with folic acid (1 mg per day) was recommended. Moreover, acetaminophen was discontinued.

In clinical and paraclinical follow-up, her signs and symptoms became better two weeks after iron therapy and bleeding time became normal ( 4 minutes and 15 seconds, IVY method), but her bleeding tendency continued. Because new history of acute severe dizziness caused her to have trouble walking, standing, or losing her balance, she was admitted to hospital for better evaluation. PTT was slightly prolonged (38.5 seconds) but PT was considerably high (21.2 seconds, INR: 2.51). As she had normal liver function tests, overdose of acetaminophen or liver disease was ruled out. A complete coagulation factor assay test was performed for her, and the results were as follows: factor IX, 24\%; factor II, $41 \%$; factor VII, 3\%; and factor X, 52\%. No inhibitors were detected. Again a detailed history was taken. She stated that for the past 6 months she had been taking many high-energy drinks and she had inadequate dietary intake. Given that she had $\mathrm{VKD}$, a course of vitamin $\mathrm{K}$ therapy was started for her in the hospital. Coagulation study results became normal after
72 hours of therapy, and she was discharged from hospital with the diagnosis of VKD. Factor assay test became normal after one week of vitamin $\mathrm{K}$ therapy, with a dosage of $5 \mathrm{mg}$ orally twice per day. Coagulation tests were repeated and the results included normal PTT, normal PT (INR: 1.01), and factor IX, 102\%; factor II, 99\%; factor VII, 97\%; and factor X, 115\% (IL ACL 7000 Coagulation Analyzer). The patient was on regular follow-up with the normal physical exam and without complaining of bleeding tendency.

\section{Discussion}

Heavy menstrual bleeding that occurs at regular intervals or at the onset of menses is often related to a bleeding diathesis and less commonly to systemic illness or structural lesions.

Given inherited bleeding disorders are found in 5 to $28 \%$ of hospitalized adolescent females with heavy menstrual bleeding; thus, these disorders should be considered in differential diagnosis $[5,6]$. Vitamin $\mathrm{K}$ acts as a cofactor in $\gamma$-carboxylation of factors II, VII, IX, and X. VKD results in reduced biological activity of these factors presenting with increases in PT or aPTT, which are corrected by vitamin K therapy [7]. Because factor VII has the shortest half-life, PT becomes prolonged first. Serum concentration of phylloquinone (vitamin K1), vitamin K1 2, 3-Epoxide (K1 O), and PIVKA-II (under carboxylated factor II) can be measured, but usually the diagnosis is clinical and by exclusion [8]. These specific vitamin $\mathrm{K}$ concentrations were not measured in our patient due to unavailability of the test in our research center.

Humans do not maintain large stores of vitamin $\mathrm{K}$ and anything that interrupts the supply from diet or gut bacteria can result in a deficiency [9]. An adult daily intake of about 100 micrograms of phylloquinone is recommended for the maintenance of hemostasis [10]. There are two kinds of combined deficiency of the vitamin K-dependent clotting factors. The first, hereditary combined deficiency of the vitamin K-dependent clotting factors is an inherited enzyme deficiency involving vitamin $\mathrm{K}$ metabolism that is an extremely rare autosomal recessive disorder in adults and is present in fewer than 30 kindred worldwide [5]. The second one is due to nutritional vitamin $\mathrm{k}$ deficiency commonly seen in neonate as hemorrhagic disease of newborns [11]. Acquired VKD in nonhospitalized adults manifesting as overt spontaneous bleeding especially menorrhagia is a rare phenomenon. Although VKD theoretically could occur in these patients, the very small daily requirement of vitamin $\mathrm{K}$ means it does not happen commonly [12].

Secondary VKD can also occur in patients undergoing major surgery or in those treated with long-term parenteral nutrition and broad-spectrum antibiotic therapy [13]. Secondary VKD is caused by the inhibition of the hepatic vitamin $\mathrm{K}$ epoxide reductase enzyme, resulting in impaired vitamin $\mathrm{K}$ recycling. Extremely high doses of vitamins $\mathrm{E}$ and A can cause VKD [14]. Secondary VKD can also occur in bulimia, those on stringent diets, and those taking anticoagulants [15].

Our patient was diagnosed with secondary VKD, resulting from inadequate nutritional intake (with a diet containing mostly energy drinks) over a period of several months, in 
addition to excessive acetaminophen intake. Energy drinks (consumed by our patients) contain amounts of vitamins $\mathrm{A}$ and $\mathrm{E}$ without vitamin $\mathrm{K}$, which can explain high daily intake relating to VKD. Physicians should keep acquired VKD in mind as a differential diagnosis of bleeding diathesis in nonhospitalized patients, especially in patients with inadequate nutritional intake. This case showed the potential for menorrhagia due to secondary VKD with use of high-energy drinks and the value of a complete and detailed history in early diagnosis.

Recognition of VKD is critical to make appropriate treatment and prevention of treatment failure, because underlying hemostatic disorder may lead to continued menorrhagia and diminished quality of life due to unnecessary gynecologic surgical interventions. We also suggest further research on contents of commercial energy drinks which affect acquired VKD.

\section{Conflicts of Interest}

The authors declare that they have no conflicts of interest regarding this paper.

\section{Authors' Contributions}

Omid Reza Zekavat and Nader Shakibazad contributed to the conception and design of the research; Sezaneh Haghpanah contributed to editing the manuscript; Seyed Javad Dehghani and Maryam Zekavat contributed to the acquisition; Gholamreza Fathpour and Nader Shakibazad contributed to drafting of the manuscript. All authors drafted the manuscript, critically revised the manuscript, agree to be fully accountable for ensuring the integrity and accuracy of the work, and read and approved the final manuscript.

\section{References}

[1] P. A. Kouides, "Bleeding symptom assessment and hemostasis evaluation of menorrhagia," Current Opinion in Hematology, vol. 15, no. 5, pp. 465-472, 2008.

[2] I. M. Nilsson, "Von Willebrand's disease-fifty years old., Acta Medica Scandinavica, vol. 201, no. 6, pp. 497-508, 1977.

[3] J. A. Bevan, K. W. Maloney, C. A. Hillery, J. C. Gill, R. R. Montgomery, and J. P. Scott, "Bleeding disorders: A common cause of menorrhagia in adolescents," Journal of Pediatrics, vol. 138 , no. 6, pp. 856-861, 2001.

[4] I. El-Hemaidi, A. Gharaibeh, and H. Shehata, "Menorrhagia and bleeding disorders," Current Opinion in Obstetrics \& Gynecology, vol. 19, pp. 513-520, 2007.

[5] N. Ö. Kanbur, O. Derman, T. Kutluk, and A. Görgey, "Coagulation disorders as the cause of menorrhagia in adolescents," International Journal of Adolescent Medicine and Health, vol. 16, pp. 183-185, 2004.

[6] R. Díaz, J. E. Dietrich, D. Mahoney, D. L. Yee, and L. V. Srivaths, "Hemostatic Abnormalities in Young Females with Heavy Menstrual Bleeding," Journal of Pediatric and Adolescent Gynecology, vol. 27, no. 6, pp. 324-329, 2014.

[7] K. L. Berkner, "Vitamin K-Dependent Carboxylation," Vitamins and Hormones, vol. 78, pp. 131-156, 2008.
[8] T. Sato, L. J. Schurgers, and K. Uenishi, "Comparison of menaquinone- 4 and menaquinone-7 bioavailability in healthy women," Nutrition Journal, vol. 11, no. 1, article no. 93, 2012.

[9] R. I. Parker, "Etiology and treatment of acquired coagulopathies in the critically ill adult and child," Critical Care Clinics, vol. 13, no. 3, pp. 591-609, 1997.

[10] M. J. Shearer, "Vitamin K in Parenteral Nutrition," Gastroenterology, vol. 137, no. 5, pp. S105-S118, 2009.

[11] Centers for Disease Control and Prevention (CDC), "Notes from the field: late vitamin $\mathrm{K}$ deficiency bleeding in infants whose parents declined vitamin $\mathrm{K}$ prophylaxis-Tennessee, 2013," Morbidity and Mortality Weekly Report, vol. 62, pp. 901902, 2013.

[12] C. Martinez, A. Katholing, K. Folkerts, and A. T. Cohen, "Risk of recurrent venous thromboembolism after discontinuation of vitamin $\mathrm{K}$ antagonist treatment: a nested case-control study," Journal of Thrombosis and Haemostasis, vol. 14, no. 7, pp. 1374$1383,2016$.

[13] C. A. Hooper, B. B. Haney, and H. H. Stone, "Gastrointestinal bleeding due to vitamin $\mathrm{K}$ deficiency in patients on parenteral cefamandole," Lancet, vol. 1, pp. 39-40, 1980.

[14] F. R. Smith and D. S. Goodman, "Vitamin A transport in human vitamin A toxicity," New England Journal of Medicine, vol. 294, no. 15, pp. 805-808, 1976.

[15] H. W. Kwon, S. H. Lee, J. E. Lee, Y. Moon, and S. K. Kim, "A Case of Acquired Transient Vitamin K Deficiency in a Teenage Girl," Clinical Pediatric Hematology-Oncology, vol. 21, pp. 33-36, 2014. 


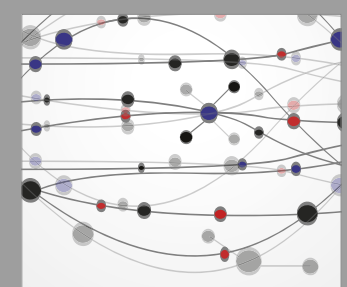

The Scientific World Journal
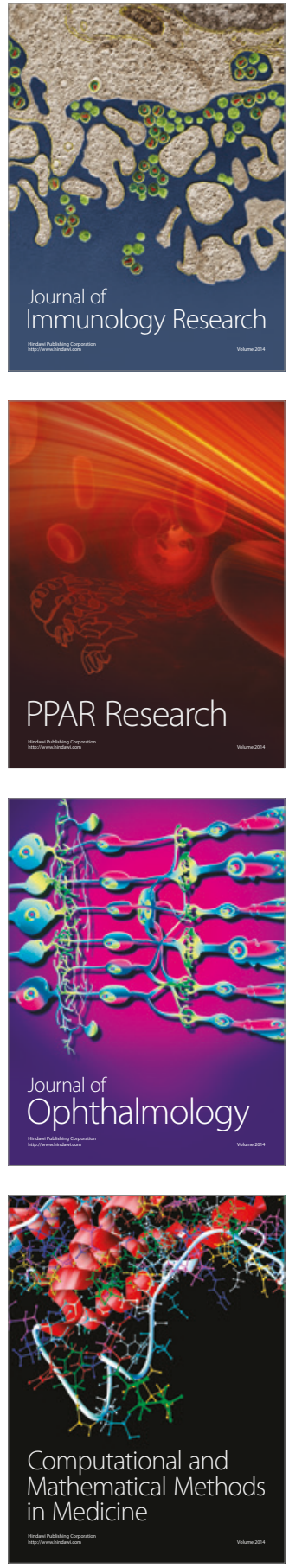

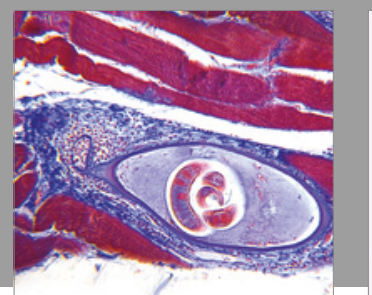

Gastroenterology Research and Practice
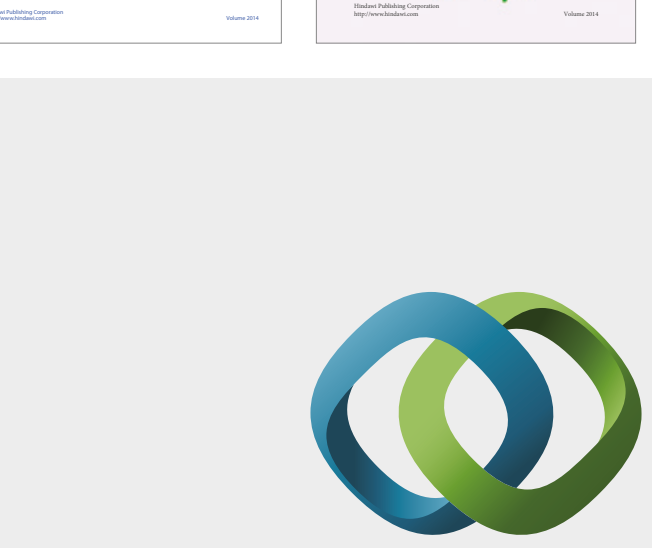

\section{Hindawi}

Submit your manuscripts at

https://www.hindawi.com
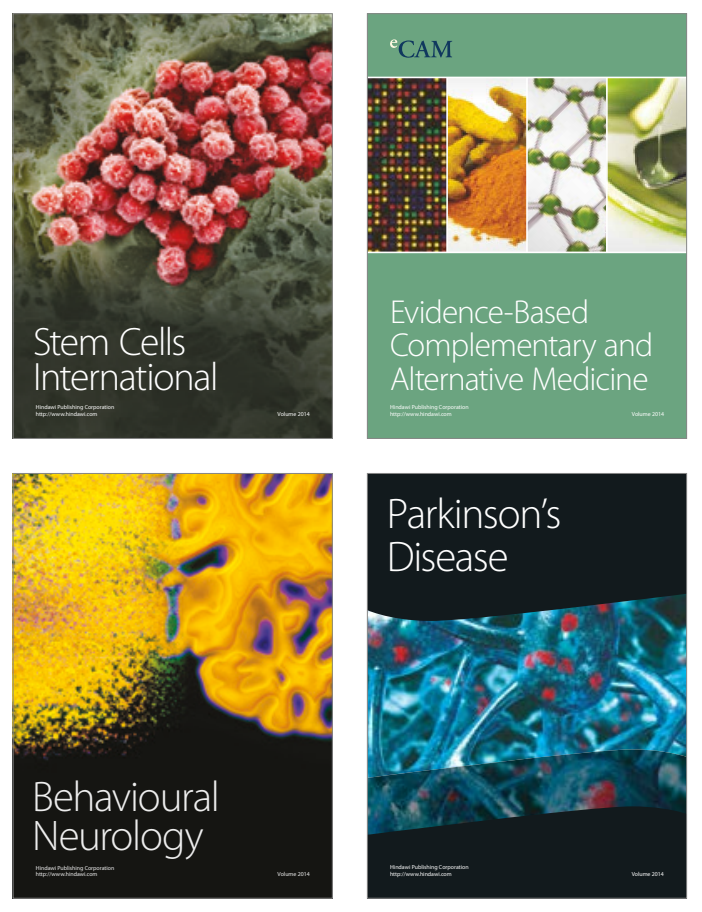
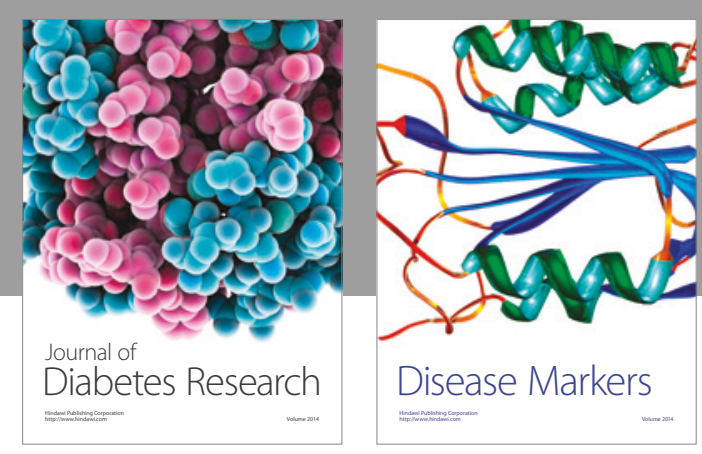

Disease Markers
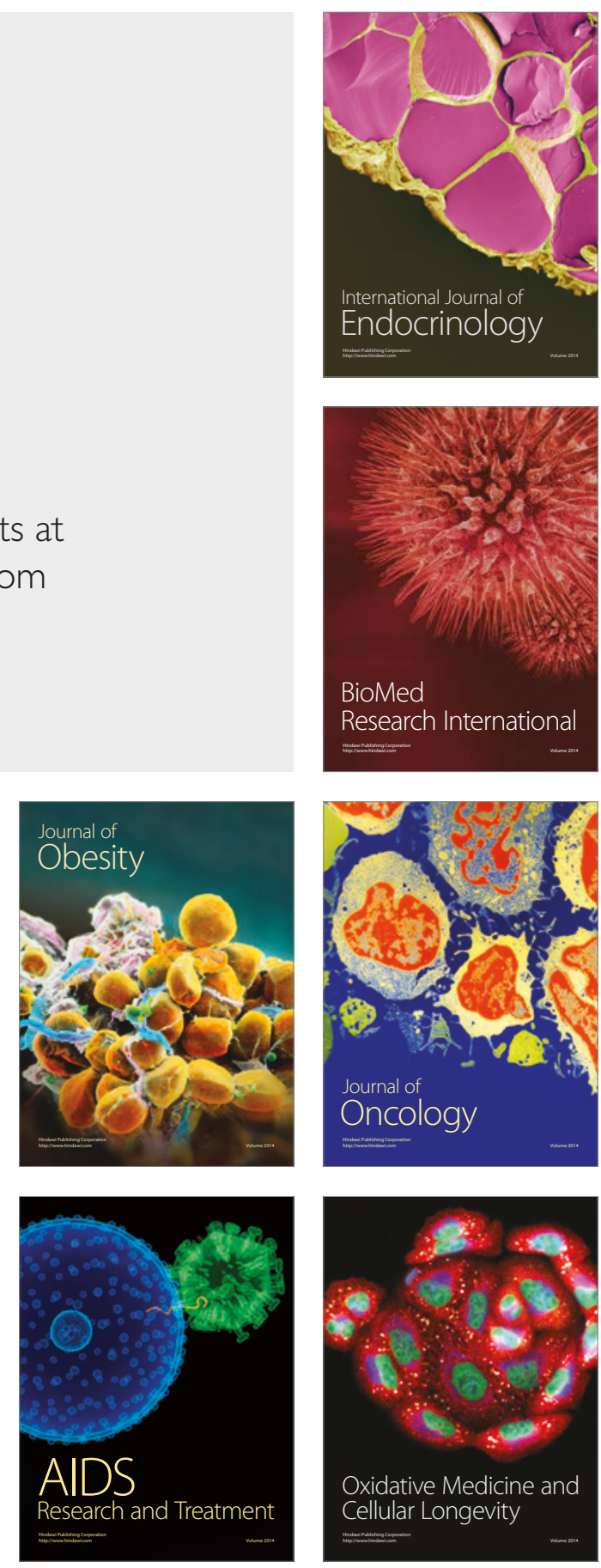